\title{
Usage of information technologies for increasing quality of transport operations
}

\author{
Mikhail Shulyak ${ }^{1}$, Oksana Pankova ${ }^{1}$, Ivan Kolesnik ${ }^{1}$, Kirill Sirovitskiy ${ }^{1}$, and Evgen Gaek ${ }^{1}$ \\ ${ }^{1}$ P. Vasilenko Kharkov National Technical University of Agriculture, 44, Alchevskykh street, \\ Kharkiv, 61002, Ukraine
}

\begin{abstract}
Among the whole cycle of interconnected technological operations for crop and livestock production, transport operations play a significant part: transportation of seed grain, forage, fertilizers, product transportation to storage and selling places. Transport operations account for $15-25 \%$ of the production cost. According to the analysis of recent research, the development and introduction of new methods for ensuring the quality and safety of production. For a tractor on transport works the task of increasing the functional precision at which it is solved its deviation from the roadway configuration (traffic corridor) during the movement is assessed. In this case the problem for short-term one-time and repeated, long-term discrete and continuous impact of the driver on the tractor steering control is solved. For these operating modes of a tractor during transport works, the methodology of functional stability providing of a hydro-level steering control is substantiated. Based on the reasoned analytical model of the tractor spin during transport works, an express method of diagnosing the technical state of the tractor steering has been elaborated. It is based on the comparison of the angular accelerations of the tractor and the steering wheel when the motion direction is changed or adjusted.
\end{abstract}

\section{Introduction}

With the increase in agricultural production and entering new markets by Ukraine, the issue of quality and environmental friendliness is becoming more and more relevant [1]. According to the analysis of recent research, the development and introduction of new methods for ensuring the quality and safety of production by means of the study of mathematically grounded models, practical measures and modern information technologies proves that without the symbiosis of science and practice it is impossible to obtain competitive products of crop sector and livestock [2-6].

Among the whole cycle of interconnected technological operations for crop and livestock production, transport operations play a significant part: transportation of seed grain, forage, fertilizers, product transportation to storage and selling places. Transport operations account for $15-25 \%$ of the production cost [7]. Tractors are mostly used for transport operations in crop and livestock production. The development of theoretical

\footnotetext{
* Corresponding author: pankova oksana@ukr.net
} 
foundations and methodological principles for efficiency improving of the technical state control of the tractor steering in transport works plays one of the most important roles in ensuring the timeliness and reliability of transport operations carrying out.

In these research works it is noted that the problem of ensuring the dynamics of mobile machinery (including tractors on transport works), in the corridor of motion, depends essentially on the technical state of the steering, the variable mass of the transport unit, the periodicity of the impact on the steering wheel, etc. Vehicles used in agricultural production for the freighting differ significantly from those used in other industries. This connects with a number of specific features: the variety of transported load, seasonality of works, urgency, overall dimensions, etc. This problem is not solved in the direction of increasing the functional precision of tractor movement at transport work.

\section{Methodology}

The task of increasing the functional precision is solved for the tractor during transport works. With it, its deviation from the desired path within the traffic corridor is estimated.

In this case the problem for short-term one-time and repeated, long-term discrete and continuous impact of the driver on the tractor steering control is solved. For these operating regimes of a tractor during transport works the methodology of functional stability providing of a hydro-level steering control is substantiated. The first type of impact is characteristic when a tractor tripping-over an obstacle, pit, etc.; the second one - with a short-term one-time impact on the steering system with maintaining its operability if the movement of the transport unit is provided in the traffic corridor, in which its diametrical deviation (output parameter) $\Delta z(t)$ from nominal value $Z_{x}$ is in the permissible range:

$$
\alpha \leq \Delta z(t) \leq \beta,
$$

where $\alpha, \beta$ are limits of the operability area: $\alpha<0, \beta>0$.

In the general case, the steering control error in the case of short-term one-time impact is a random quantity. As a result, the efficiency of this system is also a random quantity. The complete probability of realization of the assigned task by the steering control in the point of time $t$ is found as mathematical expectation of the effectiveness function:

$$
F(t)=\int_{-\infty}^{\infty} \Phi(\Delta z / t) f(\Delta z / t) d \Delta z
$$

where $\mathrm{F}(\Delta z / t)$ is probability of realization a functional task at a time $t$ by steering; $f(\Delta z / t)$ is the probability density of the steering control error at the moment $t$; $\Delta z$ is functional steering control error.

In the case of different configurations of the roadway, we can accept that the boundaries (1) of a system functionality scope in the general case are random variables with some common probability density $\omega_{t}(\alpha, \beta)$ [8-10]. Then, for all possible values of the functionality scope limits, the realization probability of target goal of the transport unit steerability (mathematical expectation of the solution of the assigned task) by steering control is written in the form:

$$
F(t)=\int_{0}^{\infty} \int_{-\infty}^{0} \int f(\Delta z / t) \omega_{t}(\alpha, \beta) d z d \alpha d \beta
$$

where $\omega_{t}(\alpha, \beta)$ - the probability density of the system in the range $\alpha$ and $\beta$. 
For the case of a short-term one-time effect on steering system, the carrying out probability by a system of an assigned task (the effectiveness function) is determined by the dependence:

$$
F(\Delta z / t)=\left\{\begin{array}{l}
F_{\alpha}(\Delta z / t)=\int_{-\infty}^{\Delta z} \omega_{t}(\alpha) d \alpha \text { at } \Delta z \leq 0 \\
F_{\beta}(\Delta z / t)=\int_{\Delta z}^{\infty} \omega_{t}(\beta) d \beta \text { at } \Delta z>0
\end{array}\right.
$$

where $\omega_{t}(\alpha), \omega_{t}(\beta)$ - accordingly, the probability density of the lower and upper limits of the operability area.

By the way of differentiation (4) by the experimental uncertainty $\Delta z$, we obtain:

$$
\omega_{t}(\alpha)=\left.\frac{d F(\Delta z / t)}{d \Delta z}\right|_{\Delta z=\alpha} ; \omega_{t}(\beta)=-\left.\frac{d F(\Delta z / t)}{d \Delta z}\right|_{\Delta z=\beta} .
$$

Analyzing dependences (4) and (5) it can be concluded that, for practical calculations, the accuracy of a transport unit movement with a short-term one-time impact on steering can be estimated with a uniform distribution of the traffic corridor limits.

The more compound interdependence between the efficiency of the steering control $\mathrm{F}(\Delta z)$ and functionality area exists in the case of short-term multiple impact. To increase the probability of solving this problem, short-term impact can be applied consistently $n$ times, at some point of time $t_{1}, t_{2}, \ldots, t_{n}$. The probability of carrying out task in each $i$-moment is equal to $\mathrm{F}\left(t_{i}+\tau_{i}\right) \approx \mathrm{F}\left(t_{i}\right)$, where $\tau_{i}$ - the action time of $i$-operation that was initiated at the moment $t_{i}$. To carrying out task it is enough that the impact on the steering solve it at least once at any $n$ moment.

If the short-term impact on the tractor's steering does not provide accuracy in the traffic corridor, then it is necessary to switch over a mode of continuous impact on the steering.

This operation mode of the transport unit is characterized when its movement on long turns. In this case, with a long impact $\tau$ on the tractor's steering from the driver's side, the system changes its state with a probability close to one, as well as in systems with prolonged discrete effects. In this case, the accuracy of the unit movement by time $\tau$ will not be equal to the motion accuracy without influence, i.e. $\mathrm{F}\left(t_{0}+\tau\right) \neq \mathrm{F}\left(t_{0}\right)$, where $-t_{0}$ the beginning of continuous control. Failure to carry out of a task that is solved at least one of the time point $\left(t_{0}, t_{0}+\tau\right)$ leads to a movement violation of the transport unit in the traffic corridor.

The problem of functional precision of tractor steering control is solved by the way of estimating the deviations (errors) of functional parameters from their calculated (nominal) values that arise under the influence of various destabilizing factors.

The level of functional precision of the steering control during the transport unit operation does not remain constant due to changes in its technical condition, disintegration, etc. In this case, during a transport unit operating, it is necessary, as a rule, to ensure minimization of the initial operation error and to maintain this parameter during prolonged operation of the transport aggregate.

On domestic and foreign tractors installed mainly hydropower steering, in which there is no mechanical connection between the steering and steerable wheels. They are connected hydraulically. The statistics of hydraulic actuator faults of tractors steering shows that according to the failures number, the failure of the main elements of the hydraulic actuator 
(hydraulic pumps, hydraulic distributors, hydraulic cylinders, etc.) prevail. Hydraulic circuit of the hydro-power steering of the tractor ensures its operation in a working order and emergency operation (volume pump exceptionally). Faults of the of the tractor hydrostatic steering elements, which lead to an increase in the leakage of the working fluid, is the main reason for the violation of the functional precision of the steering control. In this case, the functional parameters of the tractors steering control, in accordance with DSTU ISO 10998: 2013, include steerability and motion stability. When controlling steerability, the tractor's ability to react to the driver's action, aimed at preserving or changing the motion direction is estimated, and during the stability control - the preservation of a given motion direction is estimated. To take error of function of the steering control as the ability to perform the specified functions with a certain proximity degree to the ideal model, the functional error of the steering control at the current $x$ and nominal $x_{H}$ values of the functional parameters is evaluated according to:

$$
\Delta x=x-x_{H} .
$$

The probability of controlling the error of function and operability of the steering control can be estimated according to:

$$
Д=1-\left(P_{1}+P_{2}\right)
$$

where $P_{1}$ and $P_{2}$ - probability of errors of the first type (failure pass) and the second type (false refusal).

\section{Empirical results}

The probabilities of $P_{1}$ and $P_{2}$ errors depend on the partition law of values of controlled functional parameters and measurement errors, the time of the measuring process and the characteristics of the tolerance zone on the value of the measured parameter.

Steering, as a unit under test, will be operable, that is, suitable for further operation, when the measurement result satisfies the conditions:

$$
c \leq y \leq d,
$$

where $c, d$ - limits of the tolerance zone of the controlled parameter $y, 2 \delta=d-c$; $y=x_{\kappa}+\Delta x_{\kappa} ; x_{\kappa}, \Delta x_{\kappa}-$ the actual value of the monitored parameter and the error of its measurement.

Table 1. Probability of control of hydrostatic steering according to functional parameters.

\begin{tabular}{|c|c|c|}
\hline \multirow{2}{*}{ Functional parameter, Д } & Probability of error \\
\cline { 2 - 3 } & $\mathrm{P}_{1}$ & $\mathrm{P}_{2}$ \\
\hline Steerability: \\
\hline Operating fluid pressure & 0.030 & 0.015 \\
\hline Steerable wheel slip & 0.006 & 0.004 \\
\hline Steerable wheel lock at an extreme positions & 0.012 & 0.006 \\
\hline Leak resistance of the hydrovalve of the dosing pump & 0.016 & 0.008 \\
\hline Motion stability: \\
\hline Stability of uncontrolled movement & 0.014 & 0.007 \\
\hline Rate of impacts on the steering wheel & 0.003 & 0.002 \\
\hline
\end{tabular}


The sum of $P_{1}$ and $P_{2}$ indicates that in order to guarantee a given probability of control $(85 \%)$ of the tractor hydrostatic steering, the error of the technical state control of its aggregates and units according to the functional parameters should not become apparent with a probability of more than $\mathrm{P}=0,005$ (impact rates on the steering wheel) and $\mathrm{P}=0,045$ (operating fluid pressure).

The proposed methodology for controlling the error of function and operability of a hydrostatic tractor steering allows to substantiate the technology of finding faults and failures with the given probability.

Based on the reasoned analytical model of the tractor spin during transport works, an express method of diagnosing the technical state of the tractor's steering has been worked out. It is based on the comparison of the angular accelerations of the tractor and the steering wheel when the motion direction is changed or corrected.

Tractor steerability characterizes its ability to react to operator actions which are aimed at storing or changing the motion direction. The controlling impact causes the transient process from one state of the tractor balance to another, which is accompanied by a change in the traverse speed. Acceleration that arise in this case, characterize not only the tractor's steerability, but also the change in its technical condition, which is provided by the parameters instability.

It has been offered the error of function of tractor according to steerability - transfer function of the steerability:

$$
W_{k}=1-\frac{1}{K_{k}}-\frac{v}{m} \cdot \frac{d m / d t}{\dot{v}_{k}},
$$

where $K_{k}=-v_{k} / v_{c}-$ steerability coefficient; $v_{k}, v_{c}-$ the steering acceleration and acceleration of the resistance forces of the tractor withdrawal from equilibrium accordingly; $v$ - traverse speed of the tractor; $m$ - mass of the transport unit at a given point in time; $d m / d t$-rate of change of the transport aggregate mass of the variable mass.

It is proved that the tractor has perfect steerability in the conditions when $W_{k}=1$. However, this motion mode cannot be obtained, since it is impossible to get $K_{k}=\infty$ and $v / m=0$ when the tractor is moving. The approximation $W_{k}$ to a unit is possible due to providing the functional stability of the tractor steering.

An express method of assessing the technical state of a hydrostatic tractor steering has been worked out. The essence of it is to determine the turning time of the steering wheels or half-frame of the tractor on-site and to evaluate volumetric efficiency of the control system according to nomograph.

In order to determine the dependence of the volumetric efficiency of the steering control on the time of turnover from the extreme to the extreme position, it is necessary to conduct preliminarily an experimental study of determining the time dependence of the rotation on the operating fluid pressure in the steering system and obtain an approximative dependence.

Instrumentation of the express method is a measuring complex of VDVMM 4-001 was developed by the cooperation of the Kharkiv National Automobile and Highway University and the P. Vasilenko Kharkiv National Technical University of Agriculture to assess the dynamics of mobile machinery, which has been modernized by the authors for its usage in the study of tractor steerability. The measuring complex allows to carry out technical diagnostics of the hydrostatic tractor steering by the express method: the calculation of the rotation angle of the steering wheel, the angular acceleration of the tractor half-frames rotation, the transferor function of steering, the volumetric efficiency of the hydrostatic steering, the comparison of the obtained values with normative.

The express method involves the accelerated carrying out of technical diagnostics operations of the hydrostatic steering control. At the same time, as the structural parameter 
of the diagnostics the volumetric efficiency is taken, which is the determining parameter of the technical condition. And as the diagnostic parameter the angular acceleration of the tractor's turning during the transport works is taken.

The dependence of the volumetric efficiency of the hydrostatic tractor steering on the turnover time has been proved (fig. 2).

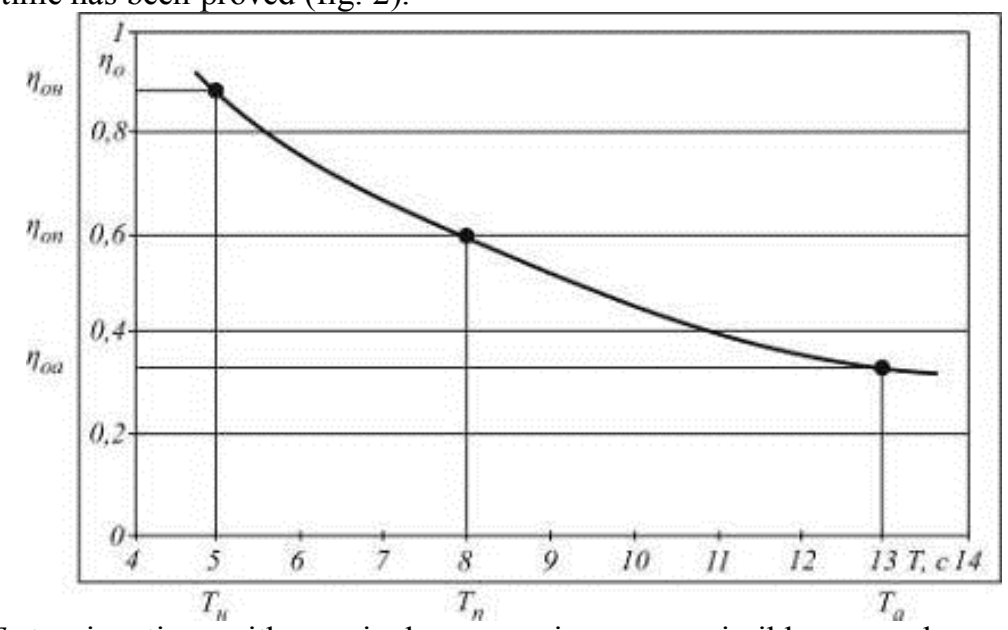

$T_{H}, T_{n}, T_{a}$ turning time with nominal $\eta_{о н}$, maximum permissible $\eta_{о \partial}$ and emergency $\eta_{о a}$ volumetric efficiency accordingly

Fig. 2. Nomogram for volumetric efficiency determination $\left(\eta_{o}\right)$ of hydrostatic tractors steering of HTZ-170 against turnover time $(T)$.

Thus, the essence of the express method for the technical state assessment of the hydrostatic power steering consist in identification, with the help of the measuring complex of the VDVMM 4-001, the turnover time of the steering wheels or half-frame of the tractor and the estimation according to the nomogram of the volumetric efficiency. It should be said that for different classes of tractors, the nomogram will be different, so the plotting of such nomograms should be carried out at the stage of research tests, with the entry of the results in the technical documentation.

\section{References}

1. V. Puzik, L. Puzik, N. Lyubymova, O. Pankova, V. Sakhatsky, IJET (UAE) 7, 507513 (2018)

2. S. Kharchenko, O. Anikeev, M. Tsiganenko, O. Kalyuzhny, KhNTUSG (MAP) 156, 174-179 (2015)

3. S. Kornienko, V. Pashenko, V. Melnik, S. Kharchenko, N. Khramov, East.-Europ. JET 5, 34-43 (2016)

4. I. Palamarchuk, O. Tsurkan, V. Palamarchuk, S. Kharchenko, East.-Europ. JET 2, 79$85,(2016)$

5. L. Tishchenko, S. Kharchenko, F. Kharchenko, V. Bredykhin, O. Tsurkan, East.Europ. JET 2, 63-69, (2016)

6. O. Nanka, O. Chalaya, S. Nagornij, O. Chalyi, East.-Europ. JET 5, 31-39, (2018)

7. E. Shubravskaya, N. Rindenko, Econ. of Ukr. 8, 77-85 (2012)

8. I. Kolesnik, UkrSRIPCT 21, 169-174 (2017)

9. M. Shulyak, SoCNC 42, 64-67 (2017)

10. M. Shulyak, EoNM 6, 99-104 (2016) 